\title{
Clonal Gene Rearrangement
}

National Cancer Institute

\section{Source}

National Cancer Institute. Clonal Gene Rearrangement. NCI Thesaurus. Code C36333.

A DNA sequence rearrangement that results in the creation of novel immunoglobulins and T-cell receptors expressed by lymphocytes. This process occurs very early in the differentiation of B- and T-lymphocytes, such that individual B- and T-cell progenitors have unique genetic profiles that are passed down to their descendants. This leads to clonal populations of lymphocytes expressing the unique proteins selected during maturation. 